\title{
A new paradigm for repair of posterior post-infarction ventricular septal defect complicated by cardiogenic shock: peripheral ECMO as a bridge to definitive operative repair
}

Vishal Khullar $^{1}$, Kukbin Choi ${ }^{1}$, and Kevin Greason ${ }^{1}$

${ }^{1}$ Mayo Clinic

November 6, 2020

\begin{abstract}
Acute post-infarction posterior ventricular septal defect (VSD) is associated with high mortality. Furthermore, patients with cardiogenic shock have been reported to have worse outcomes (1). There are conflicting reports about the optimum timing and method of surgical repair (2). Early surgical intervention has been reported to have high mortality, whereas the risk of dying in the waiting period with the medical management is also exponentially high (1). We present a case of post-infarction posterior VSD complicated by cardiogenic shock. The patient was successfully supported with peripheral veno-arterial extracorporeal membrane oxygenator as a bridge to a trans-right atrial repair.
\end{abstract}

A new paradigm for repair of posterior post-infarction ventricular septal defect complicated by cardiogenic shock: peripheral ECMO as a bridge to definitive operative repair

Running Title: Staged Post-infarction VSD Repair

Vishal Khullar, M.B.B.S, Kukbin Choi, MD, Kevin Greason, MD

Department of Cardiovascular Surgery, Mayo Clinic College of Medicine, Rochester, Minnesota.

Word count: 1,054

Corresponding author:

Vishal Khullar, M.B.B.S

Assistant Professor of Surgery

Mayo Clinic College of Medicine

200 First Street SW

Rochester MN 55905

Email:Khullar.Vishal@mayo.edu

CONFLICTS OF INTERST

None.

FUNDING

None

DATA AVAILABILITY 
Data sharing not applicable to this article as no datasets were generated or analyzed during the current study.

\section{INFORMED CONSENT, IRB APPROVAL, CLINICAL TRIAL REGISTRATION}

$\mathrm{N} / \mathrm{A}$

\section{ABRACTS}

Acute post-infarction posterior ventricular septal defect (VSD) is associated with high mortality. Furthermore, patients with cardiogenic shock have been reported to have worse outcomes (1). There are conflicting reports about the optimum timing and method of surgical repair (2). Early surgical intervention has been reported to have high mortality, whereas the risk of dying in the waiting period with the medical management is also exponentially high (1). We present a case of post-infarction posterior VSD complicated by cardiogenic shock. The patient was successfully supported with peripheral veno-arterial extracorporeal membrane oxygenator as a bridge to a trans-right atrial repair.

\section{CASE REPORT}

A previously healthy 59 years old male presented to an outside facility with an 8-day history of acute chest pain with subsequent dyspnea on exertion. Electrocardiogram showed inferior Q waves with ST segment elevation. Coronary angiogram revealed occlusion of the right coronary artery. Three drug eluting stents were placed in the right coronary artery. Subsequent echocardiogram demonstrated a large posterior ventricular septal defect (VSD) involving the papillary muscle of the tricuspid valve, severe tricuspid regurgitation, inferior wall akinesia, decreased left ventricular ejection fraction (LVEF) and mild to moderate right ventricular dysfunction (Figure 1).

Subsequently, the patient was transferred to our hospital for further management. Upon arrival, the patient was bradycardic and hypotensive. An intra-aortic balloon pump was placed, and inotropic support was started. Nevertheless, signs of multi-organ failure developed. Peripheral veno-arterial extracorporeal membrane oxygenator (VA ECMO) was placed to stabilize the patient as a bridge to operation. Since the patient's femoral arteries were severely calcified, a peripheral VA ECMO was established through the right axillary artery and the right femoral vein. Over the following 72 hours, the patient showed significant clinical and biochemical improvement. Definite surgical correction was performed.

Considering the location of the VSD, a right atrial approach was utilized. A ruptured papillary muscle to the posterior leaflet of tricuspid valve was demonstrated intraoperatively. The edge of septal defect was located approximately $2 \mathrm{~cm}$ below the tricuspid valve annulus. The defect was approximately $3 \mathrm{~cm}$ in diameter (Figure 2A). The obvious necrotic tissue was debrided. Pledget buttressed polypropylene 2-0 sutures were placed around the circumference of the ventricular septal defect with the pledgets on the left ventricular side (3). Sutures in the area near the free wall of the right ventricle were fixed from the outer wall of the right ventricle directly. A bovine pericardial patch was tailored and placed using the prefixed stiches. A running 3-0 polypropylene suture was used to secure edge of the patch (Figure 2B). A tricuspid valve replacement was also performed.

The patient was easily weaned off cardiopulmonary bypass with minimal inotropic support. Intra-operative transesophageal echocardiogram showed an intact ventricular septum and normally functioning biological prosthetic valve without significant gradient. The patient was weaned from the ventilator within 48 hours. The remainder of the post-operative course was uneventful. The patient was discharged on post-operative day 9.

\section{COMMENT}

Acute post-infarction posterior VSD causes a left to right shunt with increased pulmonary blood flow resulting in biventricular failure. An early surgical intervention without optimizing the hemodynamics can lead to high mortality (4). In addition, surgical repair of the posteriorly positioned VSD can be more challenging and is often associated with higher mortality $(1,2)$. Mechanical circulatory support in the form of a veno-arterial 
ECMO can help stabilize the patient and drain the right ventricle, thus preventing acute pulmonary edema. Moreover left ventricle can be decompressed into the right ventricle through the VSD so that left ventricular distention can also be avoided. Hence a VA ECMO can help the patient to recover from acute cardiogenic shock and give time for the VSD to fibrose so as to aid in operative repair (5).

Both trans-atrial and trans-ventricular techniques have been described in the literature to access the postinfarction posterior VSD. We feel the trans-atrial approach is associated with less bleeding complications but a higher need of tricuspid valve replacement in comparison to a trans-ventricular repair (6). In our case, the papillary muscle to the posterior leaflet of the tricuspid valve had already necrosed and ruptured, resulting in severe tricuspid regurgitation. Hence, replacement of tricuspid valve was likely going to be mandatory.

Based on our experience, we recommend that all patients in cardiogenic shock due to a post-infarct posterior VSD should be considered for VA ECMO support. Staged right atrial approach gives excellent exposure and helps avoid morbidity and mortality due to bleeding complications associated with ventriculotomy. The timing of the surgical intervention should be tailored depending upon the hemodynamic stability and presence of the cardiogenic shock.

\section{REFERENCES}

1. Cinq-Mars A, Voisine P, Dagenais F et al. Risk factors of mortality after surgical correction of ventricular septal defect following myocardial infarction: Retrospective analysis and review of the literature. Int J Cardiol 2016;206:27-36.

2. Coskun KO, Coskun ST, Popov AF et al. Experiences with surgical treatment of ventricle septal defect as a post infarction complication. J Cardiothorac Surg 2009;4:3.

3. Murashita T, Greason KL, Suri RM, Joyce DL, Stulak JM, Schaff HV. Technical modifications in the repair of acute ischemic posterior ventricular septal defect without ventriculotomy. J Card Surg 2015;30(3):233-237.

4. Arnaoutakis GJ, Zhao Y, George TJ, Sciortino CM, McCarthy PM, Conte JV. Surgical repair of ventricular septal defect after myocardial infarction: Outcomes from the society of thoracic surgeons national database. The Annals of Thoracic Surgery 2012;94(2):436-444.

5. McLaughlin A, McGiffin D, Winearls J et al. Veno-arterial ecmo in the setting of post-infarct ventricular septal defect: A bridge to surgical repair. Heart Lung Circ 2016;25(11):1063-1066.

6. Sharma V, Greason KL, Nkomo VT et al. Repair of acute inferior wall myocardial infarction-related basal ventricular septal defect: Transatrial versus transventricular approach. J Card Surg 2013;28(5):475-480. 

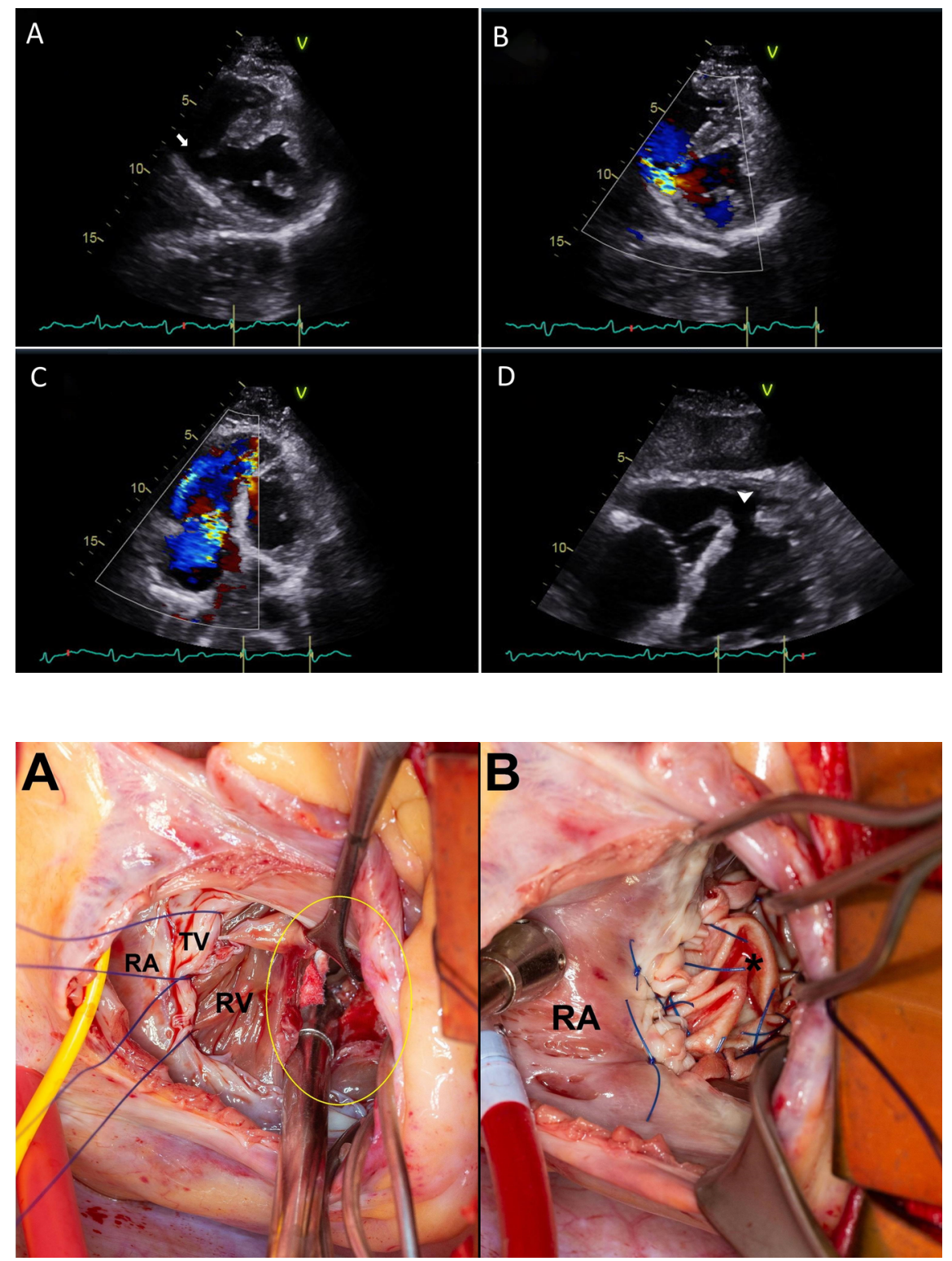